\title{
CAMBIOS EN LOS ESPACIOS FARÍNGEOS SUPERIOR MEDIO E INFERIOR CONSECUENTES A LA INSTALACIÓN DEL SIMÖES NETWORK 1 (S.N.1) EN UN GRUPO DE NIÑOS DE 7 A 10 AÑOS CON DESORDEN RESPIRATORIO RELATIVO AL SUEÑO *
}

\author{
${ }^{1}$ María Emma Zableh Solano, ${ }^{2}$ Jairo Enrique Ortiz Zambrano \\ ${ }^{1}$ Odontóloga U. Santo Tomás, Especialista en Ortodoncia U. Santo Tomás. \\ ${ }^{2}$ Odontólogo U. de Cartagena, Especialista en Ortodoncia U. Santo Tomás
}

Autor responsable de correspondencia: María Emma Zableh Solano

Correo electrónico:mezableh@gmail.com

\section{RESUMEN}

Objetivo: Evaluar los cambios en los espacios faríngeos, después de la intervención con S.N.1, en un grupo de niños con desórdenes respiratorios relativos al sueño (DRS).

Materiales y métodos: Se realizó un ensayo clínico no controlado no, con 17 individuos Clase II esquelética por retrognatismo mandibular y 5 individuos Clase I esquelética, entre 7 y 10 años de edad, con algún grado de sintomatología clínica de DRS; asistieron a la clínica de ortodoncia de la Universidad Santo Tomás Floridablanca y a una clínica privada. A cada individuo se le confeccionó e instaló un Simöes Network 1 (S.N.1). Se tomaron radiografias laterales en posiciones vertical y supina pre y post-intervención para analizar las mediciones cefalométricas de los espacios faríngeos. El índice Spearman determinó el rango de correlación de los registros ínter-evaluador. La base de datos se elaboró en Excel; se exportó al programa Statistical Product and Service Solutions (SPSS) versión 11,0 para análisis final. Se realizaron análisis descriptivos, comparaciones con chi ${ }^{2}$ para variables discretas, Prueba t de student - test para variables continuas, y análisis de varianzas (ANOVA) considerando un alfa de 0,05.

Resultados: No se encontraron diferencias entre las clases esqueléticas con relación a la obstrucción de las vías aéreas durante el sueño. Las radiografias supinas pre-intervención, mostraron disminución significativa de los espacios faríngeos. Se encontraron incrementos significativos en el espacio faríngeo superior en las tomas vertical y supina, y en el espacio medio supino post-intervención.

Conclusiones: El S.N.1 modificado es efectivo en la ampliación de la orofaringe, en la población estudiada de niños con DRS. [Zableh ME, Ortiz JE. Cambios en los espacios faríngeos superior medio e inferior consecuentes a la instalación del Simöes Network 1 (S.N.1) en un grupo de niños de 7 a 10 años con desorden respiratorio relativo al sueño. Ustasalud 2008; 7: 96 - 107]

Palabras clave: Crecimiento y desarrollo, Trastornos de la respiración, Ronquido.

\section{CHANGES IN THE SUPERIOR, MEDIUM AND INFERIOR PHARYNGEAL SPACES CONSEQUENTS TO THE INSTALLA- TION OF S.N.1 IN A GROUP OF CHIILDREN OF 7 TO 10 YEARS WITH SLEEP-REL.ATED BREATHING DISORDERS}

\section{ABSTRACT}

Objective: To evaluate the changes in the pharyngeal spaces, after the intervention with S.N.1, in a group of children with sleep-related breathing disorders (DRS).

Materials and methods: A not randomized clinical trial was done. Comparisons between a sample of 17 children skeletal class II for mandibular deficiency and 5 children skeletal class I, between 7 and 10 years of age, with some grade of clinical symptoms of DRS that were attended in the early orthodontics clinic of the Santo Tomas University in Floridablanca and in a private orthodontic clinic. For everyone of children's sample were made and installed a Simöes Network 1 (S.N.1). Lateral x-rays were taken in vertical and in supine dorsal position. Cephalometric measurements of the pharyngeal spaces were made pre and post intervention. Spearman index was used to determine the range of correlation of the registrations inter-appraiser. The database in Excel was exported to Statistical Product and Service Solutions (SPSS) version 11.0 for their final analysis. They were carried out descriptive analysis, comparisons with $c h i^{2}$ for discreet variables, t-test for continuous variables, and analysis of variances, considering an alpha of 0.05 .

Results: There was not difference among the skeletal classes with relationship to the symptoms of obstruction of the airway during the dream. Pre-intervention supine x-rays, showed a significant decrease of the pharyngeal spaces. Significative increment was observed in the superior pharyngeal space in vertical and supine position and the medium pharyngeal space in supine position, postintervention.

Conclusions: The modified S.N.1 is effective in the amplification of the oropharynx, in this children's sample with DRS.

Key words: Growth and development, Breathing dysfunctions, Snore.

Recibido para publicación: 6 de mayo de 2008. Aceptado para publicación: 18 de febrero de 2009.

* Grupo de Investigación CYDUSTA. 


\section{INTRODUCCIÓN}

La asociación entre ronquido, obstrucción de la vía aérea superior y letargo se ha reconocido desde el siglo pasado. Los Desórdenes Respiratorios Relativos al Sueño (DRS) son relativamente comunes durante la niñez. Tienen una larga escala de signos y síntomas desde ronquido primario hasta Síndrome de Apnea Obstructiva del Sueño (SAOS), una entidad asociada a ronquido con disrupciones repetitivas de la vía aérea durante el sueño. Como la mayoría de los síntomas tienen lugar durante el sueño, pueden pasar desapercibidos fácilmente. ${ }^{1-10}$ Se considera que el SAOS está subdiagnosticado en la primera y segunda infancia y a menudo es la causa de desórdenes escolares o de conducta. ${ }^{11-19}$

A este respecto, es importante anotar que el ronquido primario es un síntoma que acompaña a la obstrucción respiratoria alta; sin embargo, no todos los que padecen esta sintomatología, necesariamente padecen el SAOS. ${ }^{1-13}$

La etiología más común del SAOS en niños es la hipertrofia del tejido adenoideo faríngeo ${ }^{1-10}$ También, se ha reconocido que la obstrucción de la vía aérea superior en los niños puede evolucionar a problemas sistémicos (hipertensión, desórdenes cardiacos) y que esta situación puede revertirse mediante amigdalo-adenoidectomía. ${ }^{1-13}$

Existe un número limitado de estudios que investigan el predominio del ronquido y del SAOS en los niños. Más del $7 \%$ al $10 \%$ de la población infantil ronca regularmente. ${ }^{1-13}$ Esta condición puede alterar la calidad de vida tanto del paciente como la de su familia. ${ }^{1,10,13,18}$ Aunque algunos desórdenes respiratorios ocurren únicamente durante el descanso nocturno, virtualmente todos los desórdenes respiratorios empeoran durante el sueño comparativamente a la vigilia. El enfoque de los estudios de investigación está dirigido a establecer las diferencias entre los disturbios respiratorios de adultos y niños desde la perspectiva del desarrollo. ${ }^{3-14}$ Debido al proceso de crecimiento y desarrollo, el SAOS puede afectar a la niñez de una manera específica no encontrada en adultos.

La fisiopatología y la etiología de los desórdenes respiratorios relacionados al sueño están inadecuadamente entendidos. El complejo proceso de obstrucción de la vía aérea durante el sueño es un fenómeno dinámico que no puede ser explicado únicamente por factores mecánicos. Se ha establecido que esta obstrucción tiene implicaciones directas tanto en la fisiología del sueño como en la fisiología respiratoria. ${ }^{4-20}$
La hipertrofia del tejido adenoideo faríngeo, puede ser registrada mediante la medición de los espacios de la vía aérea, en un cefalograma lateral de cabeza. ${ }^{14,21-23}$ Los resultados de la medición cefalométrica pueden revelar que existen diferencias estadísticamente significativas en la comparación de los valores de los espacios faríngeos en niños con hipertrofia de tejido adenoideo y niños normales. Dado que existen pocos estudios en la población colombiana y que no existe ningún estudio en la población santandereana, los resultados deben ser comparados con estudios realizados en otras poblaciones.

El tratamiento de estos procesos de obstrucción respiratoria alta, debe involucrar un equipo interdisciplinario que esté destinado a identificar el problema, determinar la etiología y establecer los parámetros de tratamiento precisos para los diversos grados de complejidad que ella presenta. ${ }^{2-6,8-10}$

En la actualidad, no están absolutamente claros y definidos los factores predisponentes, desencadenantes, coadyuvantes y perpetuantes de la obstrucción respiratoria alta. Así mismo, no se han establecido mecanismos de prevención y tratamiento. La etiología multifactorial explica la complejidad en la selección de las alternativas de tratamiento, y no siempre los procedimientos quirúrgicos proporcionan curación y estabilidad de los resultados. ${ }^{1-11}$ La selección de la adenotonsilectomía como método de tratamiento no siempre es curativa. ${ }^{4,20}$

Recientes publicaciones apuntan las directrices de tratamiento del SAOS leve y moderado hacia el uso de reposicionadores mandibulares tanto en la población adulta como infantil. ${ }^{24}$ Estudios actuales han puesto en evidencia la eficacia de las aparatologías intraorales en el control de las obstrucciones respiratorias. ${ }^{25-34}$ El Simöes Network 1 (S.N.1) es un aparato ortopédico funcional de los maxilares que puede ser confeccionado con el objeto de ubicar la mandíbula y la lengua en una posición más anterior, incrementando el espacio aéreo de la faringe..$^{35}$

Este trabajo de investigación permite evaluar los cambios producidos en los espacios faríngeos, después de la intervención con S.N.1, en un grupo de niños que presentan desórdenes respiratorios relativos al sueño. Responde a la hipótesis de que este reposicionador mandibular, incrementa los espacios faríngeos superior, medio e inferior tanto en posición cefálica vertical como en supina dorsal. Establece la relevancia de la intervención temprana en el tratamiento de algunos de los sig- 
nos y síntomas presentes en el SAOS. Además, aumenta el conocimiento en esta área y ofrece una guía para el diagnóstico y tratamiento de este trastorno respiratorio. Para tal fin, se efectuó un ensayo clínico no controlado, no aleatorizado.

\section{MATERIALES Y MÉTODOS}

Se seleccionaron 22 niños y niñas con síntomas de obstrucción respiratoria que asistieron a las clínicas de ortodoncia de la Universidad Santo Tomás en Floridablanca y a una clínica de carácter privado. Todos tenían indicación de tratamiento de ortodoncia temprana. La muestra fue dividida en dos grupos de acuerdo con el tipo de maloclusión que presentaban: Clase II esquelética por retrognatismo mandibular y Clase I esquelética.

\section{Criterios de inclusión:}

Grupo 1

- Niños con edades entre los 7 y 10 años en los que es común la hipertrofia de los tejidos adenoideos.

- Historia de ronquido primario de más de seis meses de evolución

- Signos y síntomas de respiración mixta o alergia respiratoria

- Clase II esquelética por retrognatismo mandibular. Ángulo ANB $>$ de $4^{\circ}$

Grupo 2

- Niños con edades entre los 7 y 10 años.

- Con historia o sin historia de ronquido primario de más de seis meses de evolución.

- Con signos y síntomas de respiración mixta o antecedentes de alergia respiratoria.

- Clase I esquelética. Ángulo ANB de 2 a $4^{\circ}$

\section{Criterios de exclusión (para ambos grupos):}

- Niños Clase I o Clase II esquelética que no requieran tratamiento de ortopedia maxilar.

- Niños a los que les habían practicado la adenotosilectomía.

- Niños no sindrómicos con macroglosia (el volumen lingual podría agravar la obstrucción de la vía aérea).

- Individuos con síndromes que incluyan alteraciones de tamaño mandibular o lingual.

A todos los individuos les fueron tomadas impresiones en alginato, que fueron vaciadas en yeso tipo III. Además, se tomaron registros del cambio de postura mandibular en cera rosada para bases: para el grupo 1 en clase I canina y para el grupo 2 en contacto de incisivos en determinada área (D.A). ${ }^{35}$

En los modelos de yeso y con el registro de cambio de postura mandibular, se confeccionó el reposicionador mandibular S.N.1 a cada uno de los participantes (Figuras 1 a 5). El Simoes Network 1 es un aparato ortopédico funcional bimaxilar que consta de dos placas de acrílico, una es el segmento inferior que incluye dos tubos telescópicos de 0.40 ' de diámetro interno, donde se insertan dos respectivos arcos dorsales (alambre S.S. 0,36' hard) provenientes de la placa superior con el objeto de reposicionar anteriormente la mandíbula y no permitir su retroceso. La asociación entre tubos telescópicos y arcos dorsales permite el movimiento protrusivo y lateroprotrusivo mandibular..$^{35}$ Una de las recomendaciones de un estudio previo a este con otro tipo de reposicionador, fue la de implementar o diseñar futuros aparatos que además de cambiar la posición mandibular, permitan movimientos mandibulares, para evitar el cansancio muscular. ${ }^{36}$

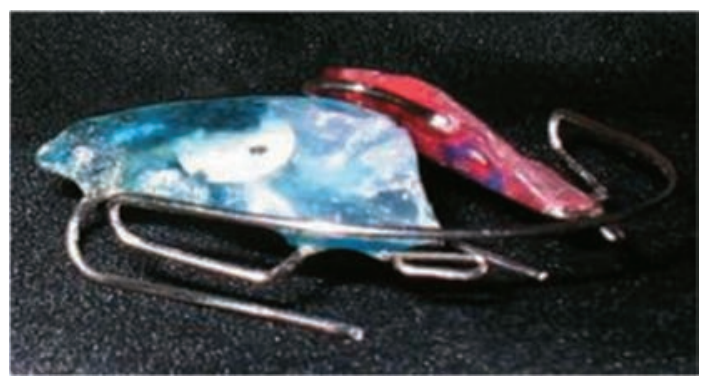

Figura 1. Segmento superior, nótese los arcos dorsales.

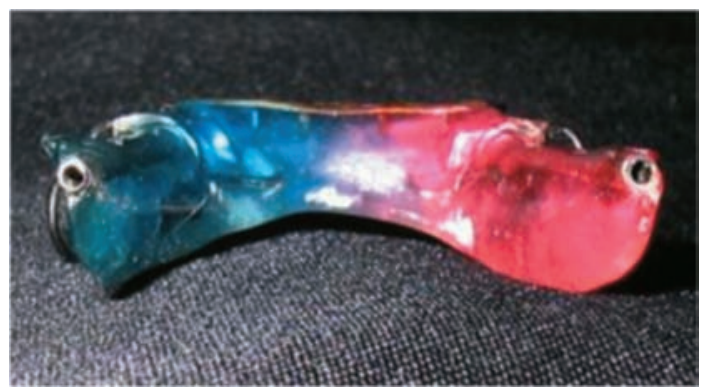

Figura 2. Segmento inferior (vista posterior). Se aprecian los tubos.

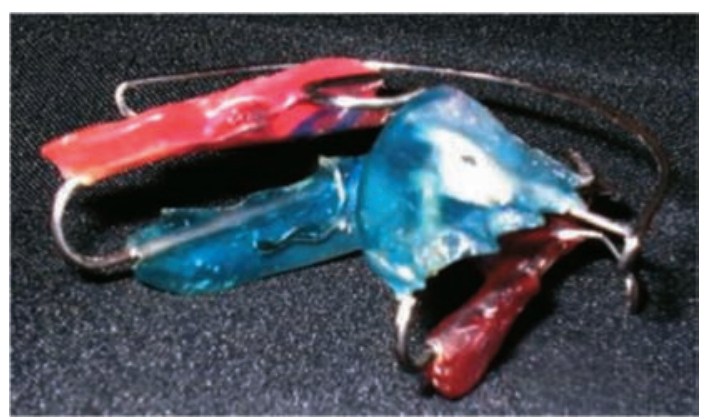

Figura 3. Aparato ensamblado. 


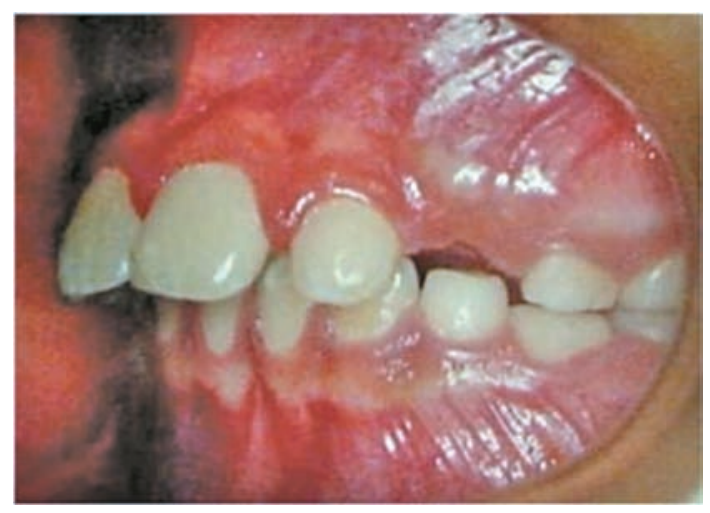

Figura 4. Oclusión izquierda pre intervención.

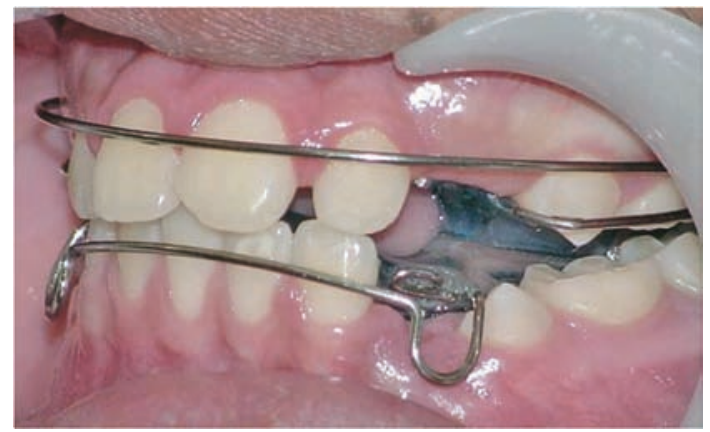

Figura 5. Oclusión izquierda post intervención.

Todos los individuos del grupo 2 (clase I esquelética) eran tratados por una deficiencia transversal (apiñamiento) y una sobremordida vertical aumentada, mediante aparatología ortopédica funcional pistas Planas para clase I. ${ }^{35-37}$ A estos aparatos se les realizó una modificación que consistió en insertar tubos telescópicos en la base acrílica inferior y arcos dorsales (S.S. hard calibre $0,40^{\prime}$ ) en la base acrílica superior. Esta modificación se realizó con el objeto de convertir las pistas Planas en S.N.1 modificado, durante la toma de los registros radiográficos.

Posteriormente se tomaron radiografias de perfil en posición vertical (sentado) y en posición supina dorsal con y sin aparato (Figura 6). Mediante la medición cefalométrica de los espacios faríngeos antes y después de la instalación del reposicionador se pudo determinar su porcentaje de incremento.

Se registraron las variables cefalométricas de los espacios faríngeos superior (SPAS), medio (MAS) e inferior (IAS), según el protocolo de Ozbek, Lowe y colaboradores y el valor del ángulo ANB. ${ }^{24}$ Se incluyeron los datos de género, edad y sintomatología de obstrucción respiratoria: ronquido, bruxismo nocturno y sueño no reparador.

Se realizaron análisis descriptivos, comparaciones con chi cuadrado para variables discretas, t-test para variables continuas, y análisis de varianza (ANOVA) considerando un alfa de 0,05 . Se estratificaron los niños por género, por clasificación esquelética y por grupos de edad (7 años y 8 a 10 años).

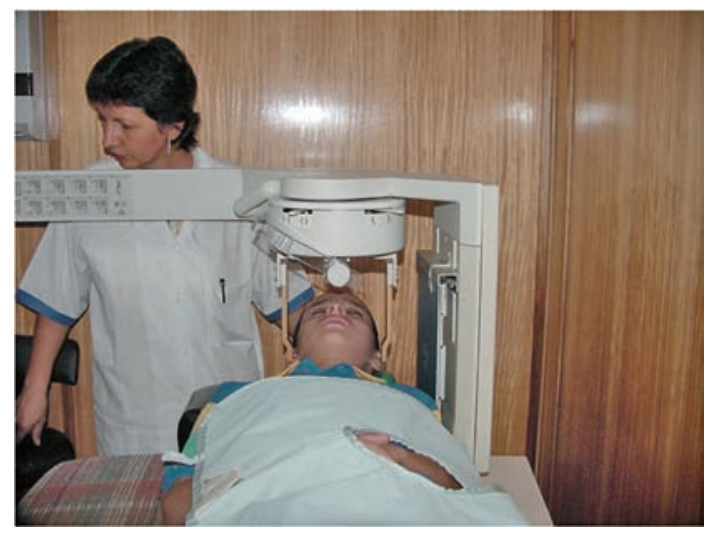

Figura 6. Paciente en posición supina dorsal.

Las diferencias en los espacios faríngeos superior, medio e inferior fueron comparadas mediante la aplicación del test estadístico de t-Student pareada, antes y después de la intervención en cada grupo para detectar cambios estadísticamente significativos. Además se compararon los promedios de los espacios aéreos entre los dos grupos experimentales. Se establecieron comparaciones con los estándares establecidos por Bolton en las radiografias en posición vertical. ${ }^{38,39}$

\section{RESULTADOS}

El 54.5\% (12) de la muestra fueron niñas. La moda en la población completa fue de 10 años y la media de 9.09 años (dt: 1.109). Con relación a los síntomas de obstrucción durante el sueño, se encontraron $14(63.6 \%)$ individuos con ronquido, 19 (86.4\%) con bruxismo (86.4\%) y $15(68.2 \%)$ con sueño no reparador.

Diecisiete (77.3\%) individuos presentaban clase II esquelética según el valor del ángulo ANB mayor de $4^{\circ}$ en la cefalometría diagnóstica inicial. No se encontraron diferencias entre las clases esqueléticas I y II con relación a la sintomatología de obstrucción de las vías aéreas durante el sueño.

El rango de correlación entre las medidas registradas por tres operadores fue de 0.93 a 1 (correlación de Spearman, $\mathrm{p}<.0001$ ).

No se encontraron diferencias de género en las medidas previas a la intervención ni en los cambios en los espacios faríngeos pre y post intervención. Las medidas tomadas, sus medias, intervalos de confianza del $95 \%$ de las diferencias y test estadístico son reportados en la Tabla 1. 
Tabla 1. Medias de las medidas tomadas, sus diferencias y desviación típica, test estadístico y significancia.

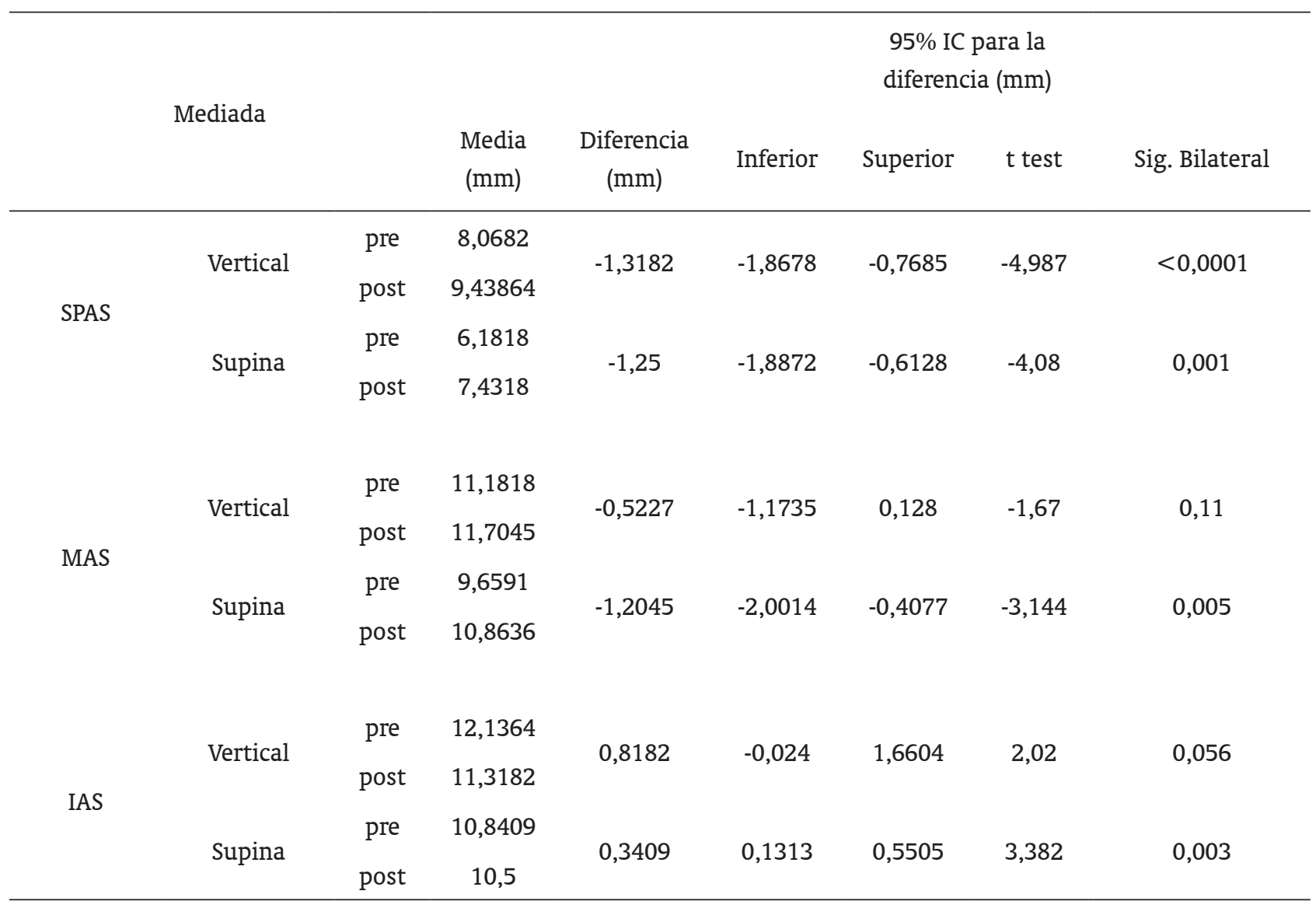

Nota: Valores promedios de todos los datos. Grados de libertad $=21$.

$n=22$

En la población completa se encontró un incremento significativo en el SPAS post intervención en posición vertical (diferencia de $1.409 \mathrm{~mm}$ con un $95 \%$ de confianza entre 0.7986 y $2.0196 \mathrm{~mm}$ ) $\mathrm{y}$ en posición supina (diferencia de $1.250 \mathrm{~mm}$, intervalo de confianza del $95 \%$ de la diferencia 0.6128 -1.8872). También se encontró incremento significativo en el MAS en posición supina dorsal (diferencia $1.2045 \mathrm{~mm}$, IC $95 \% 0.4077$ a 2.0014 $\mathrm{mm}$ ) en el grupo general.

En el análisis del grupo Clase I esquelética, se encontraron diferencias estadísticamente significativas del IAS en posición vertical, entre las radiografías pre y post intervención reportándose disminución en este espacio aéreo después de la intervención (1.9 mm IC 95\% de la diferencia de 0.3541 a $3.4459 \mathrm{~mm}$ ) (Tabla 2).

En el grupo de Clase II esquelética, se encontraron incrementos significativos en las mediciones radiográficas pre y post-intervención de SPAS y MAS en posición vertical y supina (Tabla 3). El aumento del espacio faríngeo superior en la toma vertical fue, en promedio, de $1.6166 \mathrm{~mm}$ y esta diferencia tuvo un rango de 0.0 a $5.0 \mathrm{~mm}$ (Figuras 3 y 4). En la toma en posición supina la diferencia fue en promedio de $1.6176 \mathrm{~mm}$ con un rango entre $0 \mathrm{~mm}$ y $4.0 \mathrm{~mm}$. En la toma en posición supina del espacio faríngeo medio, se evidenció un aumento promedio de $1.5 \mathrm{~mm}(\mathrm{dt}=1.94454)$ post-intervención con valores de incremento del espacio hasta $6 \mathrm{~mm}$ y disminución del mismo hasta un milímetro.
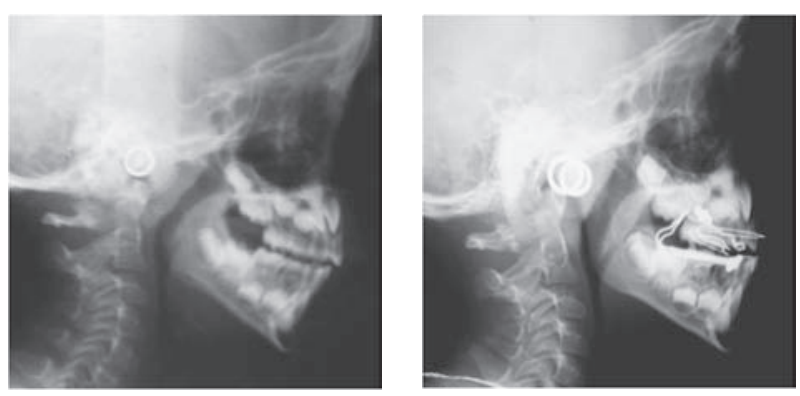

Figura 7 y 8. Radiografías de perfil en posición vertical pre y post-intervención. Nótese la ampliación de los espacios faríngeos posterior a la intervención. 
Tabla 2. Clase esquelética I. Medidas y sus medias, diferencias y sus intervalos de confianza del 95\%, test estadístico y su significancia.

\begin{tabular}{|c|c|c|c|c|c|c|c|c|}
\hline & \multirow[b]{2}{*}{ Medida } & & \multirow[b]{2}{*}{$\begin{array}{c}\text { Media } \\
(\mathrm{mm})\end{array}$} & \multicolumn{4}{|c|}{$\begin{array}{c}\text { 95\% IC para la } \\
\text { diferencia }(\mathrm{mm})\end{array}$} & \multirow[b]{2}{*}{ Sig. Bilatera } \\
\hline & & & & $\begin{array}{l}\text { Diferencia } \\
\text { (mm) }\end{array}$ & Inferior & Superior & $\mathrm{t}$ test & \\
\hline \multirow{3}{*}{ SPAS } & \multirow[t]{2}{*}{ Vertical } & pre & 6,9 & \multirow[t]{2}{*}{$-0,7$} & \multirow[t]{2}{*}{$-2,19$} & \multirow{2}{*}{0,79} & \multirow[t]{2}{*}{$-1,3$} & \multirow[t]{2}{*}{0,263} \\
\hline & & post & 7,6 & & & & & \\
\hline & Supina & pre & 5,6 & 0 & $-1,16$ & 1,16 & 0 & 1,000 \\
\hline \multirow{4}{*}{ MAS } & \multirow{2}{*}{ Vertical } & pre & 12,6 & \multirow{2}{*}{0,2} & \multirow{2}{*}{$-1,84$} & \multirow{2}{*}{2,24} & \multirow{2}{*}{0,27} & \multirow{2}{*}{0,799} \\
\hline & & post & 12,4 & & & & & \\
\hline & \multirow{2}{*}{ Supina } & pre & 11,6 & \multirow{2}{*}{$-0,2$} & \multirow{2}{*}{$-0,75$} & \multirow{2}{*}{0,35} & \multirow{2}{*}{-1} & \multirow{2}{*}{0,374} \\
\hline & & post & 11,8 & & & & & \\
\hline \multirow{4}{*}{ IAS } & \multirow{2}{*}{ Vertical } & pre & 13,7 & \multirow{2}{*}{1,3} & \multirow{2}{*}{$-0,774$} & \multirow{2}{*}{3,34} & \multirow{2}{*}{1,7} & \multirow{2}{*}{0,152} \\
\hline & & post & 12,4 & & & & & \\
\hline & \multirow{2}{*}{ Supina } & pre & 13,8 & 19 & 035 & 344 & 241 & (207 \\
\hline & & post & 11,9 & & 0,35 & 3,44 & 3,41 & $0,0<t$ \\
\hline
\end{tabular}

Nota: Alfa 0.05. Grados de libertad $=4$.

$n=5$

Tabla 3. Clase esquelética II. Medias de las medidas tomadas, sus diferencias y desviación típica, test estadístico y significancia.

\begin{tabular}{|c|c|c|c|c|c|c|c|c|}
\hline & \multirow[b]{2}{*}{ Medida } & & \multirow[b]{2}{*}{$\begin{array}{l}\text { Media } \\
(\mathrm{mm})\end{array}$} & \multirow[b]{2}{*}{$\begin{array}{l}\text { Diferencia } \\
(\mathrm{mm})\end{array}$} & \multicolumn{2}{|c|}{$\begin{array}{l}\text { 95\% IC para la } \\
\text { diferencia (mm) }\end{array}$} & \multirow[b]{2}{*}{ t test } & \multirow[b]{2}{*}{ Sig. Bilateral } \\
\hline & & & & & Inferior & Superior & & \\
\hline \multirow{4}{*}{ SPAS } & \multirow{2}{*}{ Vertical } & pre & 8,3529 & \multirow{2}{*}{$-1,6166$} & \multirow{2}{*}{$-2,3606$} & \multirow{2}{*}{$-0,9047$} & \multirow{2}{*}{$-4,81$} & \multirow{2}{*}{$<0,0001$} \\
\hline & & post & 9,9706 & & & & & \\
\hline & \multirow{2}{*}{ Supina } & pre & 6,3529 & \multirow{2}{*}{$-1,6176$} & \multirow{2}{*}{$-2,3189$} & \multirow{2}{*}{-09164} & \multirow{2}{*}{$-4,89$} & \multirow{2}{*}{$<0,0001$} \\
\hline & & post & 7,9706 & & & & & \\
\hline \multirow{4}{*}{ MAS } & \multirow{2}{*}{ Vertical } & pre & 10,7647 & \multirow{2}{*}{$-0,7356$} & \multirow{2}{*}{$-1,4516$} & \multirow{2}{*}{$-0,0189$} & \multirow{2}{*}{$-2,18$} & \multirow{2}{*}{0,045} \\
\hline & & post & 11,5 & & & & & \\
\hline & \multirow{2}{*}{ Supina } & pre & 9,0882 & \multirow{2}{*}{$-1,5$} & \multirow{2}{*}{$-2,4998$} & \multirow{2}{*}{$-0,5002$} & \multirow{2}{*}{$-3,18$} & \multirow{2}{*}{0,006} \\
\hline & & post & 10,5882 & & & & & \\
\hline \multirow{4}{*}{ IAS } & \multirow{2}{*}{ Vertical } & pre & 11,6765 & \multirow{2}{*}{0,6765} & \multirow{2}{*}{$-0,3476$} & \multirow{2}{*}{1,7005} & \multirow{2}{*}{1,4} & \\
\hline & & post & 11 & & & & & 0,0 \\
\hline & Sunina & pre & 9,9706 & -0 9412 & -24204 & 05381 & -135 & 0196 \\
\hline & supinda & post & 10,9118 & 20,012 & $-2,4204$ & 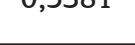 & כב, 1- & 0,170 \\
\hline
\end{tabular}

Nota: Alfa 0.05. Grados de libertad $=16$

$n=17$ 
Al correlacionar los valores del ángulo ANB con las diferencias obtenidas en las mediciones post intervención, se halló una correlación en la medida del IAS de -0.487 (Rho de Spearman, $\mathrm{p}=0.022$ ). Al analizar la gráfica obtenida se evidencia una tendencia clara al aumento del espacio faríngeo en los pacientes Clase II esquelética (Figura 9).

\section{Diferencia en los espacios faríngeos de la posi- ción vertical a supina pre y post-intervención para los pacientes clase II intervenidos}

Se encontraron diferencias significativas en las medias de las medidas SPAS, MAS, IAS pre-intervención al pasar de posición vertical a supina, disminuyéndose las dimensiones del SPAS en promedio de 2 $\mathrm{mm}(\mathrm{dt}=1.18585, \mathrm{p}=0.000)$, para MAS en promedio de $1.6765 \mathrm{~mm}(\mathrm{dt}=1.40247, \mathrm{p}=0.000)$, $\mathrm{y}$ disminución del IAS en promedio $1.7059 \mathrm{~mm}(\mathrm{dt}=$ 2.62237, $\mathrm{p}=0-016)$.

Post-intervención, la medida SPAS se disminuye de vertical a supina en promedio $2 \mathrm{~mm}$ (dt $=1.64886, \mathrm{p}$ $=0,000)$. MAS presentó disminución post-interven- ción de 0.9118 ( $\mathrm{dt}=1.32565, \mathrm{p}=0.012$ ). El IAS no expresó disminución significativa post intervención $(\mathrm{p}=0.853)$.

\section{Análisis de las variables con referencia a los patrones establecidos por Bolton}

Se estratificaron los individuos por grupos de edad para poder compararlos con los patrones establecidos por el estudio de Bolton de la siguiente manera: los pacientes de siete años se compararon con los patrones para SPAS, MAS y IAS de Bolton reportados para 6 años y los pacientes de 8 , 9, y 10 años se compararon con los patrones para 9 años.

Tres individuos del total de la muestra tenían 7 años de edad y 19 individuos se encontraban en el intervalo de 8 a 10 años (86.4\%) de la población (Tabla 4). Para los pacientes de 7 años no hubo diferencia significativa con los valores previamente estipulados con los registros de los estándares de Bolton en ninguna de las tomas radiográficas verticales, no pre, no post.

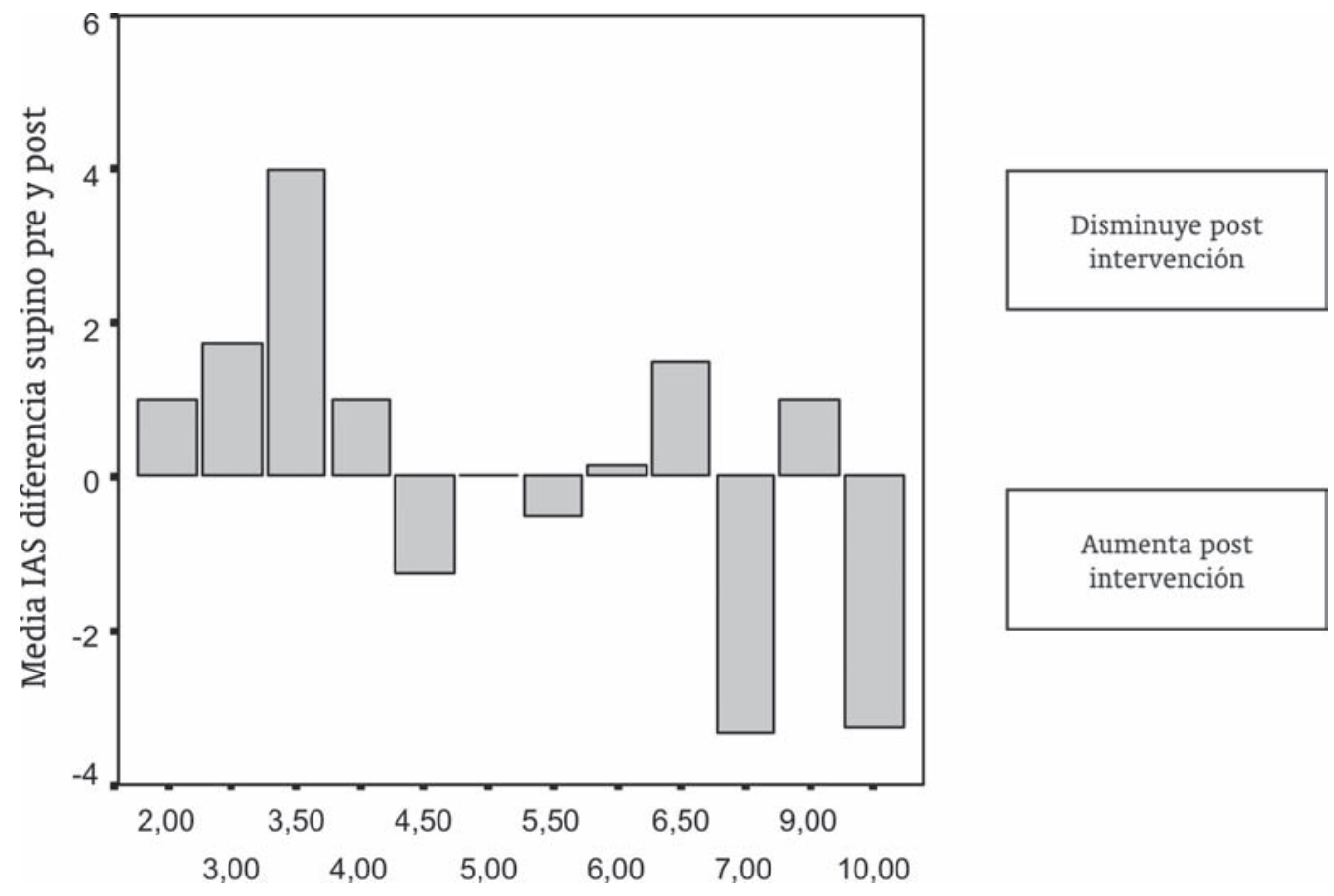

Figura 9. Relación ANB y diferencia espacio IAS

Los registros de los pacientes de 8 a 10 años incluidos en esta muestra, al ser comparados con los patrones estándares de Bolton para nueve años, presentaron diferencias significativas en la medición del SPAS en posición vertical previo a la intervención siendo ésta $2.8158 \mathrm{~mm}$ de menor tamaño que el patrón ( $p$ de 0.003 ) y el SPAS medido en posición vertical, posterior a la intervención no tuvo diferencia con el patrón. En la radiografía previa y posterior, la medida del MAS en posición vertical reveló un espacio faríngeo significativamente más amplio en esta muestra al ser comparado con los patrones estándares de Bolton y las diferencias, con un $95 \%$ de confianza, se ampliaron com- 
parativamente $0.4498 \mathrm{~mm}$ para el límite inferior y $0.4449 \mathrm{~mm}$ para el superior. La medida del IAS en posición vertical previa a la intervención fue mayor comparada con el patrón de los estándares de Bolton, en un promedio de $2.1364 \mathrm{~mm}(\mathrm{p}=0.001)$, con valores de diferencia entre $0.9218 \mathrm{~mm}$ y 3.3509 $\mathrm{mm}$ con un $95 \%$ de confianza. Las mediciones en las radiografias posteriores a la intervención, aunque fueron significativamente más amplias que el patrón de los estándares Bolton, disminuyeron en comparación con la toma previa al tratamiento.

No se encontraron diferencias estadísticamente significativas en cuanto al género y los estratos.

Tabla 4. Estrato de niños de 8 a 10 años comparados con los estándares de 9 años de Bolton.

\begin{tabular}{ccccccccc}
\hline & Medida & & $\begin{array}{c}\text { Media de } \\
\text { la muestra }\end{array}$ & Diferencia & Inferior & Superior & t test & $\begin{array}{c}\text { Sig. } \\
\text { Bilateral }\end{array}$ \\
\hline \multirow{2}{*}{ SPAS } & Vertical & pre & 8,1842 & $-2,8158$ & $-4,5559$ & $-1,0757$ & $-3,4$ & 0,003 \\
& & post & 9,3947 & $-1,6053$ & $-3,4381$ & 0,2276 & $-1,84$ & 0,082 \\
& & & & & & & & \\
\multirow{2}{*}{ MAS } & Vertical & pre & 11,5789 & 1,3789 & 0,3463 & 2,4116 & 2,805 & 0,012 \\
& & post & 12,0263 & 4,2263 & 0,7961 & 2,8565 & 3,724 & 0,002 \\
& & & & & & & & \\
\multirow{2}{*}{ IAS } & Vertical & pre & 12,1364 & 2,1364 & 0,9218 & 3,3509 & 3,658 & 0,001 \\
& & post & 11,3182 & 1,3182 & 0,2862 & 2,3502 & 2,656 & 0,015 \\
\hline
\end{tabular}

Nota: Grados de libertad $=18$.

\section{DISCUSIÓN}

Muchos autores han enfatizado la importancia de relacionar la posición corporal con los cambios que acontecen en la vía aérea faríngea. De acuerdo con los resultados obtenidos, en la población objeto de este estudio, se disminuyen las dimensiones de la vía aérea faríngea al pasar de posición vertical a supina dorsal. Este hallazgo ha sido ampliamente reportado en adultos. ${ }^{40-45}$ Sin embargo, en esta investigación se pudo observar un aumento del IAS, en seis individuos clase II ( $27 \%$ de la muestra). Resultados similares han sido reportados por Brander y colaboradores, quienes encontraron un incremento significativo del área faríngea inferior en posición supina en 72 hombres adultos obesos y no obesos con desorden respiratorio relativo al sueño. ${ }^{46}$

No obstante, reportan los valores del ángulo ANB 1 \pm 3 en no obesos y $2 \pm 3$ en obesos, sin especificar el porcentaje de individuos clase II. ¿Cómo explicar un comportamiento distinto al restante $73 \%$ de la población estudiada? Tsuchiya y colaboradores, lograron mediante un análisis, identificar diferentes subtipos de pacientes apneicos adultos, utilizaron la electromiografia. ${ }^{47}$ Partinen y colaboradores lograron subdividir la población de pacientes apneicos estudiados, de acuerdo con diferencias en masa corporal y posición del hueso hioides entre otros. ${ }^{48}$ De acuerdo con las evidencias obtenidas en estos estudios de adultos, se puede llegar a pensar que probablemente también existan diferentes subtipos de DRS durante el crecimiento y desarrollo. Estos subgrupos podrían representar diferentes entidades, con distintas consideraciones terapéuticas, en las que la aparatología intraoral bien podría no ser siempre efectiva.

Las radiografias en posición supina de este estudio fueron tomadas de acuerdo con el protocolo de Pae, Lowe y colaboradores, en una confortable y natural posición de dormir. ${ }^{40}$ Durante ese proceso de relajación, se observó un descenso de la mandíbula (Figura 10). Este fenómeno ha sido estudiado por varios autores. ${ }^{49-51}$ En sus resultados describen una nueva dimensión vertical de importancia clínica y funcional, denominada dimensión vertical de reposo neuromuscular (8 a $10 \mathrm{~mm}$ de distancia interoclusal), que corresponde con la verdadera longitud de reposo de los músculos elevadores mandibulares, con mínima actividad electromiográfica tónica de ellos. Manns y Díaz reportaron que los mecanismos de control de la postura mandibular pueden ser no nerviosos o pasivos (propiedades viscoelásticas musculares, espacio de Donders y fuerzas gravitacionales) y nerviosos o activos (tono muscular)..$^{52} \mathrm{Un}$ estudio de la actividad tónica de los músculos ele- 
vadores mandibulares, reveló una disminución de la actividad electromiográfica de posición vertical a supina. La mayor actividad en $90^{\circ}$ es debida a las fuerzas gravitacionales que estimulan la actividad neuromuscular. La disminución en $0^{\circ}$ (acostado) se debe a que las fuerzas gravitacionales actúan en ángulo recto en relación con las fibras elevadoras, determinando una menor actividad de los husos neuromusculares. ${ }^{53}$

El creciente uso de reposicionadores mandibulares para tratar obstrucciones orofaríngeas durante el sueño, hace indispensable determinar su eficacia inmediata y sus efectos posteriores. En el presente estudio, el S.N.1 fue efectivo en la ampliación de la orofaringe en ambos grupos experimentales. Estudios a corto y a largo plazo han demostrado la efectividad de los reposicionadores mandibulares en la ampliación de los espacios faríngeos en adultos. ${ }^{25-34,55}$ Lowe, realizó un estudio comparativo entre 13 distintas aparatologías; las medidas lineales cefalométricas demostraron incrementos de alrededor del $100 \%$ en los tres espacios faríngeos utilizando el aparato de Herbst. Concluye que el Jasper Jumper no es capaz de contrarrestar la fuerza gravitacional de la mandíbula en posición supina, ni siquiera adicionando elásticos verticales. ${ }^{31}$ En otro estudio, el mismo autor reconstruye en tres dimensiones la vía aérea mediante escanografia, antes y después de la inserción de un reposicionador mandibular, observó una elongación anteroposterior de la totalidad del tubo respiratorio después de la intervención. ${ }^{55}$

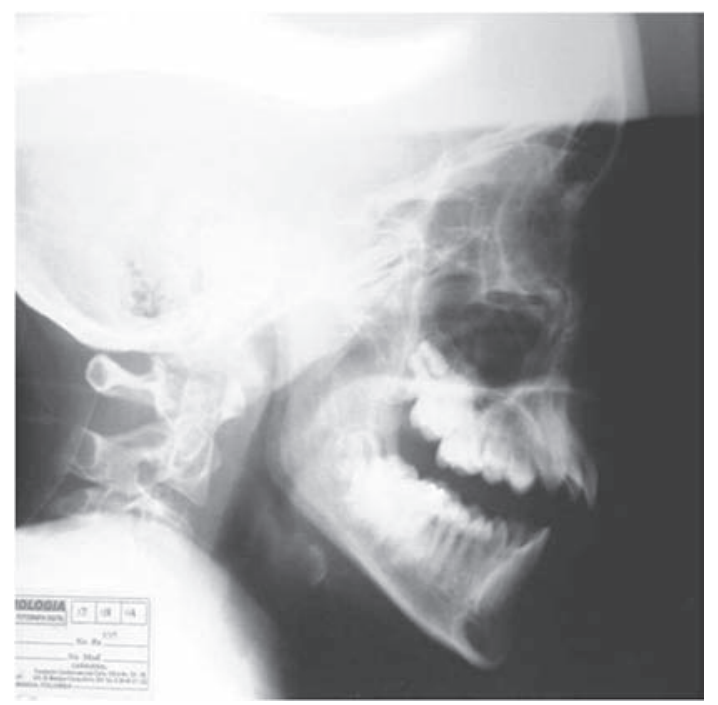

Figura 6. Radiografia de perfil en posición supina dorsal preintervención.

Los resultados de esta investigación revelaron diferencias entre ambos grupos experimentales. Probablemente sean debidas a distintos comportamientos neuromusculares propios para individuos Clase I o Clase II con obstrucción respiratoria. Pae y colaboradores resaltan que pacientes con SAOS severo, no obesos, pueden tener la altura facial inferior corta, la mordida profunda y un retrognatismo mandibular; sin embargo, las variables cefalométricas sugieren que la relación sagital maxilo-mandibular puede no ser la causa del trastorno obstructivo, pero sí la mordida profunda; recomiendan tener en cuenta este hallazgo a la hora de la selección del tratamiento. ${ }^{56}$ Cabe anotar que dentro del procedimiento de este trabajo investigativo, el cambio de postura mandibular del aparato en el grupo Clase I esquelética fue de tipo vertical (contacto de incisivos en D.A.) a diferencia del grupo de Clase II esquelética donde se realizó un cambio de postura mandibular de tipo horizontal, para corregir el retrognatismo mandibular. En los individuos Clase I esquelética se tomó la decisión de no realizar cambio de postura mandibular de tipo horizontal porque la relación oclusal una vez insertado el aparato, pasaría a ser Clase III. Por su parte, Rose y colaboradores concluyeron que el uso de aparatologías intraorales a largo plazo puede causar efectos dentales colaterales significativos, como vestibuloversión de incisivos, y alteraciones en la relación oclusal, entre otros. ${ }^{25}$

En las tomas radiográficas posteriores a la intervención, realizadas en este estudio, se encontró una disminución del SPAS $(2 \mathrm{~mm}, \mathrm{dt}=1.64886, \mathrm{p}=$ 0.000 en promedio) y del MAS $(0.9118 \mathrm{dt}=1.32565$, $\mathrm{p}=0.012$ ), al pasar de posición vertical a una supina dorsal, en el grupo Clase II esquelética. Posiblemente, la disminución del SPAS y del MAS, sea debida a la dimensión vertical de reposo neuromuscular ya mencionada, descrita por Manns y otros autores, como resultado de la relajación muscular en posición supina dorsal. En consecuencia, el arco dentario inferior queda desarticulado del segmento inferior del aparato. ${ }^{49-53}$ En la literatura revisada no se encontró que otros autores hayan reportado esta limitación; posiblemente, utilizaron aparatos rígidos con mecanismos retentivos para evitar la desarticulación del aparato.

Al correlacionar los valores del ángulo ANB con las diferencias obtenidas en las mediciones post-intervención, se observó una correlación en la toma IAS de -,487 (Rho de Spearman, $\mathrm{p}=0.022$ ). Al analizar la gráfica obtenida se observo una tendencia clara al aumento de este espacio faríngeo a medida que se incrementa el valor del ángulo ANB (Figura 3). Existen en la literatura gran cantidad de referencias acerca de la asociación entre retrognatismo mandibular y disminución de la dimensión orofaríngea tanto en adultos como en niños; algunos de ellos superan la barrera de la bidimensionalidad radiográfi- 
ca y emplean tomografias, resonancia magnética y video-endoscopia. ${ }^{21-24,42,45,56-68}$

Özbek y colaboradores, publicaron, en 1998, los resultados de un estudio realizado en niños y niñas Clase II $(n=20)$, cuyo propósito fue probar la eficiencia de la aparatología funcional en la corrección del retrognatismo mandibular. Incluyeron como grupo control 15 niños que habían participado en un estudio longitudinal de crecimiento. El promedio de edad fue 11.3 años. ${ }^{24}$ Registraron las variables cefalométricas ANB, SPAS, MAS e IAS. Sus resultados evidenciaron incrementos significativos en las dimensiones de la orofaringe, especialmente en los casos de mayor ANB pre-tratamiento al ser comparadas con el grupo control. Mediante estudios electromiográficos, varios autores han establecido, diferencias en los patrones neuromusculares del complejo hioideo y del músculo geniogloso en pacientes apneicos. ${ }^{50-59}$ Dichas diferencias posiblemente puedan darle explicación a los hallazgos encontrados en este y en los demás estudios referenciados; sería importante seguir investigando al respecto.

Es importante anotar que en esta investigación no se encontraron diferencias entre Clase I y Clase II esquelética con relación a la sintomatología de obstrucción de las vías aéreas durante el sueño: bruxismo, ronquido y sueño no reparador. Estos signos han sido ampliamente reportados en estudios de desórdenes del sueño en adultos, donde establecen claramente una asociación entre obesidad, redundancia en tejidos blandos y retrognatismo mandibular (clase II esquelética)., ${ }^{2,7}$ Posiblemente, las diferencias entre adultos y niños radiquen en los factores etiológicos, dado que la obstrucción rino y orofaríngea en niños se encuentra estrechamente asociada a la hipertrofia del tejido adenoideo. ${ }^{1-15,20,69,70,71}$

El grupo Clase II esquelética, presentaba disminución en la vía aérea faríngea en comparación con los registros del estudio longitudinal "Estándares de Bolton". ${ }^{38,39}$ Las comparaciones se realizaron con las tomas verticales pre-intervención. Los resultados no expresan diferencias con los registros para los 7 años, pero si se encontró en el grupo etáreo de 8 a 10 años, una disminución de $2.8 \mathrm{~mm}(\mathrm{p}=0.003)$ en promedio para el SPAS en el $86,4 \%$ de la muestra analizada. Kulnis, Nelson y colaboradores en un estudio cefalométrico en 28 niños roncadores, con longitud efectiva mandibular corta vs. 28 no roncadores, reportaron las mayores diferencias en los espacios faríngeos superior y medio, siendo éstos significativamente menores en el grupo de niños roncadores. ${ }^{72}$

El SPAS en posición vertical en el grupo Clase II, posterior a la inserción del aparato no tuvo diferencias con los registros del estudio de Bolton. Esto podría significar que con el cambio de postura del grupo Clase II, las dimensiones de la vía aérea alcanzan los mismos valores que los estándares. Dentro de la bibliografía estudiada, no se encontraron estudios longitudinales que incluyan registros de la vía aérea faríngea realizados en poblaciones colombianas. Un estudio realizado en Turquía, comparativo entre los Estándares de Bolton y una población de niños Clase II esquelética por retrognatismo mandibular, estableció diferencias estadísticamente significativas. Previo a la intervención con un activador de crecimiento mandibular, las dimensiones de los tres espacios faríngeos fueron significativamente menores en los individuos Clase II. Dos años después de usar el activador, los resultados evidenciaron valores cercanos a los Estándares Bolton. ${ }^{24}$

Este estudio contó con un tamaño de muestra inadecuado que no permitió realizar una aleatorización. En consecuencia, se pudieron generar sesgos de selección. Se requieren nuevas investigaciones que determinen los valores cefalométricos normales para la población colombiana.

Este es el primer estudio realizado en población santandereana, en individuos en crecimiento, que reúne diagnóstico y tratamiento de los trastornos obstructivos de la vía aérea alta, relativos al sueño. Al estimar el porcentaje de niños con alta sospecha de apnea, reportado en el estudio de Blanco, Santos y colaboradores en una población santandereana ${ }^{73}$ y las complicaciones sistémicas a largo plazo de estos trastornos respiratorios, ${ }^{1-9}$ se considera que los resultados de este estudio son de carácter relevante, de gran aplicabilidad clínica y excelente proyección.

\section{BIBLIOGRAFÍA}

1. Hui S, Wing KY. Obstructive sleep apnea syndrome in children. Hong Kong Med J 1997; 3: 419 - 426.

2. Bower CM, Gungor A. Pediatric Obstructive Sleep Apnea Syndrome. Otolaryngol Clin North Am 2000; 33: 49 - 75.

3. Marcus CL. Sleep-disordered breathing in children. Am J Respir Crit Care Med 2001; 164: 16 -30.

4. Nieminen P, Löppönen T, Tolonen U, Lanning P, Knip M, Löppönen $\mathrm{H}$. Growth and biochemical markers of growth in children with snoring and obstructive sleep apnea. Pediatrics 2002; 109: 55

5. Carroll JL, Mccolley SA, Marcus CL, Curtis S, Loughlin GM. Inability of clinical history to distinguish primary snoring from obstructive sleep apnea syndrome in children. Chest 1995; 108: 610 - 618.

6. Coleman J. Sleep Studies: Current Techniques and Future Trends. Otolaryngol Clin North Am 1999; 32: 195- 210.

7. Bahammam A, Kryger M. Decision making in obstructive sleep-disordered breathing. Putting it all together. Clin Chest Med 1998; 19: 87 - 97. 
8. Darrow DH, Weiss DD. Management of sleep-related breathing disorders in children. Head Neck Surg 2002; 13: $111-118$

9. Daniel Y, Goh T, Galster P, Marcus CL. Sleep architecture and respiratory disturbances in children with obstructive sleep apnea. Am J Respir Crit Care Med 2000; 162: 682 - 686.

10. Redline SP, Tishler V, Schluchter M, Aylor J, Clark K, Graham G. Risk factors for sleep-disordered breathing in children. Am J Respir Crit Care Med 1999; 159; 1527 - 1532.

11. Owens J, Opipari L, Nobile C, Spirito A. Sleep and daytime behavior in children with obstructive sleep apnea and behavioral sleep disorders. Pediatrics 1998; 102: 1178 1184.

12. Brown KA. What we don't know about childhood obstructive sleep apnoea. Paediatr Anaesth 2001; 11: 385 - 389.

13. Davidson Ward SL, Marcus CL. Obstructive sleep apnea in infants and young children. J Clin Neurophisiol 1996; 13: 198 - 207.

14. Ali NJ, Pitson DJ, Stradling JR. Snoring, sleep disturbance and behaviour in 4-5 year olds. Arch Dis Child 1993; 68: $360-366$.

15. Gozal D, Wang M, Pope DW Jr. Objective sleepiness measures in pediatric obstructive sleep apnea. Pediatrics 2001; 108: 693 - 697

16. Chervin RD, Archbold KH, Dillon JE, Panahi P, Pituch KJ, Dahl RE, Guilleminault C. Inattention, hyperactivity, and symptoms of sleep-disordered breathing. Pediatrics 2002; 109: 449 - 456.

17. Morelli, A, Ladan S, Ducharne FM, Brouillette RT. Can sleep and wakefulness be distinguished in children by cardiorespiratory and videotape recordings? Chest 1996; 109: 680 - 687.

18. Sivan Y, Kornecki A, Schonfeld T. Screening obstructive sleep apnoea syndrome by home videotape recording in children. Eur Respir J 1996; 9: 2127 - 2131.

19. Lamm C, Mandeli J, Kattan M. Evaluation of home audiotapes as an abbreviated test for obstructive sleep apnea syndrome (OSAS) in children. Pediatr Pulmonol 1999; 27: $267-272$.

20. Gislason T, Benediktsdottir B. Snoring, apneic episodes, and nocturnal hypoxemia among children 6 months to 6 years old. Chest 1995; 107: 963 - 966.

21. Acebo C, Millman R, Rosenberg C, Caballo A. Sleep, breathing, and cephalometrics in older children and young adults. Part I. Chest 1996; 109: $664-672$.

22. Millman R, Acebo C, Rosenberg C, Carskadon M. Sleep, breathing, and cephalometrics in older children and young adults. Part II. Chest 1996; 109: $673-679$.

23. Zucconi M, Caprioglio A, Strambi L F, Oldani A, Castronovo C, Smirme S, Craneofacial modifications in children with habitual snoring and obstructive sleep apnoea: A case control study. Eur Respiratory J 1999; 13: 411 - 417.

24. Özbek MM, Memikoglu UT, Gögen H, Lowe A, Baspinar E. Oropharyngeal airway dimensions and functional-orthopedic treatment in skeletal class II cases. Angle Orthod 1998; 68: $327-336$

25. Rose EC, Staats R, Virchow C, Jonas IE. Occlusal and skeletal effects of an oral appliance in the treatment of Obstructive Sleep Apnoea. Chest 2002; 122: 871 - 877.

26. Clark GT, Arand D, Chung E, Tong D. Effect of anterior mandibular positioning on obstructive sleep apnoea. Am Rev Respir Dis 1993; 147: 624 - 629.
27. Schmidt-Nowara W. Recent developments in oral appliance therapy of sleep disordered breathing. Sleep Breath 1999; 3: 103 - 106.

28. Schmidt-NowarA W, Lowe A, Wiegand L, Cartwright R, Perez-Guerra F, Menn S. Oral appliances for the treatment of snoring and obstructive sleep apnea: a review. Sleep 1995; 18: 501 - 510.

29. Friedlander AH, Friedlander IK, Progel MA. Dentistry's role in the diagnosis and co-management of patients with sleep apnea/hypopapnea syndrome. Br Dent J 2000; 189: 76 - 80 .

30. Schmidt-Nowara W, Lowe A, Wiegand L, Cartwright R, Pérez-Guerra F, Menn S. Oral appliances for the treatment of snoring and obstructive sleep apnea: A review. Sleep 1995; 18: 501 - 510.

31. Lowe A. Dental Appliances for the Treatment of Snoring and Obstructive Sleep Apnea. En: Kryger MH, Roth T, Dement WC. Eds. Philadelphia: WB Saunders, 1994: 722 -735 .

32. Pancer J. Evaluation of variable mandibular advancement appliance for treatment of snoring and sleep apnea. Chest 1999; 116: 1511 - 1518.

33. Johal A. Current principles in the management of obstructive sleep apnea with mandibular advancement appliances. Br Dent J 2001; 190: 532 - 536.

34. Liu Y. Effects of a mandibular repositioner on obstructive sleep apnea. Am J Dentofacial Orthop 2000; 118: 248 256.

35. Simöes WA. Ortopedia Funcional de los Maxilares vista a través de la RNO. Tomo II, Caracas, Isaro, 1988, p. 96 $-97$.

36. Barba A, Barrios L, Otero LM, Rodríguez JR. Efectividad del aparato A.R.E.M.U.S. en el tratamiento del S.A.O.S. [Trabajo de Grado] Bucaramanga: Universidad Santo Tomás; 2003.

37. Planas P. Rehabilitación Neuro-Oclusal. 2da Ed. Barcelona, Masson-Salvat, 1994, p. 50, 164-169.

38. Broadbent BH, Broadbent BH Jr, Golden WH. Bolton Standards of Dentofacial development growth. St. Luois, C.V. Mosby, 1975.

39. Taylor M, Hans MG, Strohl KP, Suchitra NS, Broadbent HB. Soft tissue growth of the oropharynx. Angle Orthod 1996; 66: 393 - 400 .

40. Pae EK, Lowe AA, SasakI K, Price C, Tsuchiya M, Fleetham JA. A cephalometric and electromyographic study of upper airway structures in the upright and supine positions. Am J Orthod Dentofacial Orthop 1994; 106: 52 - 59.

41. TakahashI S, Ono T, Ishiwata Y, Kuroda T. Effect of changes in the breathing mode and body position on tongue pressure with respiratory-related oscillations. Am J Orthod Dentofacial Orthop 1999; 115: 239 - 246.

42. Hiyama $\mathrm{S}$, Ono $\mathrm{T}$, Ishiwata $\mathrm{Y}$, Kuroda $\mathrm{T}$. Effects of mandibular position and body posture on nasal patency in normal awake subjects. Angle Orthod; 2002; 72: 547 - 553.

43. Otsuka R, Ono T, Ishiwata Y, Kuroda T. Respiratory-related genioglossus electromyographic activity in response to head rotation and changes in body position. Angle Orthod 2000; 70: $63-69$.

44. Brander PE, Mortimore IL, Douglas NJ. Effect of obesity and erect/supine posture on lateral cephalometry: relationship to sleep-disordered breathing. Eur Respir J 1999; 13: $398-402$ 
45. Ono T, Lowe AA, Ferguson KA, Fleetham JA. Associations among upper airway structure, body position, and obesity in skeletal Class I male patients with obstructive sleep apnea. Am J Orthod Dentofacial Orthop 1996; 109: $625-634$.

46. Kajaste S, Brander PE, Telakivi T, Partinen M, Mustajoki P. A cognitive-behavioral weight reduction program in the treatment of obstructive sleep apnea syndrome with or without initial nasal CPAP: a randomized study. Sleep Med 2004; 5: 125 - 131.

47. Tsuchiya M, Lowe AA, Pae EK, Fleetham JA. Obstructive sleep apnea subtypes by cluster analysis. Am J Orthod Dentofacial Orthop. 1992; 101: 533 - 542.

48. Partinen M, Guilleminault C, Quera-Salva MA, Jamieson A. Obstructive sleep apnea and celphalometric roentgenograms: the role of anatomic upper airway abnormalities in the definition of abnormal breathing during sleep. Chest 1988; 93: 1199 - 1205.

49. Manns A, Miralles R, Palazzi C. EMG, bite force, and elongation of the masseter muscle under isometric voluntary contractions and variations of vertical dimension. J Prosthet Dent 1979; 42: $674-682$.

50. Manns A, Miralles R, Guerrero F. The changes in electrical activity of the postural muscles of the mandible upon varying the vertical dimension. J Prosthet Dent 1981; 45: $438-445$.

51. Rugh JD, Drago CJ. Vertical dimension: a study of clinical rest position and jaw muscle activity. J Prosthet Dent 1981; 45: $670-675$.

52. Manns A, Díaz G. Sistema Estomatognático. Facultad de Odontología Universidad de Chile 1995. p. 162 - 164.

53. Miralles R, Manns A, Guerrero F. Study of EMG postural activity in mandibular muscles at different body positions. IRCS Medical Science 1981; 8: 122 - 123

54. McGown AD, Makker HK, Battagel JM. Estrange PR. Long -term use of mandibular advancement splints for snoring and obstructive sleep apnoea. Eur Respir J 2001; 17: $462-614$.

55. Lowe A, Fleetham J, AdachI S, Ryan F. Cephalometric and computed tomographic predictors of obstructive sleep apnea severity. Am J Orthod 1995; 107: 589 - 595.

56. Pae EK, Ferguson KA. Cephalometric characteristics of nonobese patients with severe OSA. Angle Orthod 1999; 69:: $408-412$.

57. Hilloowala RA, Trent RB, Gunel E, Pifer RG. Proposed cephalometrics diagnosis for osteogenic obstructive sleep apnea: The mandibular / Pharyngeal ratio. Cranio 1999 Oct; $17: 280-8$

58. Lowe AA, Ózbek M, Miyamoto K, Pae EK, Fleetham JA. Cephalometric and demographic characteristics of obstructive sleep apnea: An evaluation with partial least squares analysis. Angle Orthod 1997; 67: 143 - 151.

59. Pae, E-K, Lowe, AA, Fleetham JA. A role of pharyngeal length in obstructive sleep apnea patients. Am J Orthod Dentofac Orthop 1997; 111: 12 - 17.

60. Lowe AA, Ono T, Ferguson KA, Pae EK, Ryan CF, Fleetham JA. Cephalometric comparisons of craniofacial and upper airway structure by skeletal subtype and gender in patients with obstructive sleep apnea. Am J Orthod Dentofacial Orthop 1996;110: 653 - 664

61. Powell N, Riley R, Troell R, LI K, Brumen M. Radiofrequency volumetric tissue reduction of the palate subjects with sleep disordered breathing. Clinical Investigations. 1998; 113: 1163 - 1174 .
62. Sakakibara H, Tong M, Matsushita K, Hirata M, Konishi Y, Cephalometric abnormalities in non-obese patients with obstructive sleep apnoea. Eur Respir J 1999; 13: 403 410.

63. Prachartam N, Nelson S, Hans M, Broadbent H, Redline S, Rosenberg C, Cephalometric assessment in obstructive sleep apnea analysis. Am J Orthod 1996; 109: 410 - 419.

64. Arens R, McDonough JM, Aaron M, Hernandez ME, Maislin G, Schwab RJ, Pack AI. Linear dimensions of the upper airway structure during development. Am J Respir Crit Care Med 2002; 165: 117- 122.

65. Arens R , McDonough AM, Corbin NK, Rubin ME, Carroll A I, Pack J, Liu Y, Udupa JK. Upper airway size analysis by magnetic resonance imaging of children with obstructive sleep apnea syndrome. Am J Respir Crit Care Med 2003; 167: $65-70$.

66. Filho DI, Ravell DB, Ravell RB, Castro Monteiro L, GandinI LG Jr. A comparison of nasopharyngeal endoscopy and lateral cephalometric radiography in the diagnosis of nasopharyngeal airway obstruction. Am J Orthod Dentofacial Orthop 2001; 120: 348 - 352.

67. Pae EK, Lowe AA, Sasaki K, Price C, Tsuchiya M, Fleetham JA. A cephalometric and electromyographic study of upper airway structures in the upright and supine positions Am J Orthod Dentofacial Orthop 1994; 106: 52 - 59.

68. Otsuka R, Ono T, Ishiwata Y, Kuroda T. Respiratory-related genioglossus electromyographic activity in response to head rotation and changes in body position. Angle Orthod 2000; 70: $63-69$.

69. Schechter MS; Section on Pediatric Pulmonology, subcommittee on Obstructive Sleep Apnea Syndrome. Technical report: diagnosis and management of childhood obstructive sleep apnea syndrome. Pediatrics 2002; 109: e69.

70. Brunetti L, Rana S, Lospalluti ML, Pietrafesa A, Francavilla R, Fanelli M, Armenio L. Prevalence of obstructive sleep apnea syndrome in a cohort of 1207 children of southern Italy. Chest 2001; 120: 1930 - 1935.

71. Guilleminault C, Pelayo R, Leger D, Clerk A, Bocian RC. Recognition of sleep disordered breathing in children. Pediatrics 1996; 98: 871 - 872.

72. Kulnis R, Nelson S, StrohL K, Hans M. Cephalometric assessment of snoring and nonsnoring children. Chest 2000; 118: 596 - 603.

73. Blanco M, Santos MT, Madrigal LC, Camargo DM. Prevalencia de alta sospecha del síndrome de apnea obstructiva del sueño en preescolares de Bucaramanga. Ustasalud Odontología 2003; 2: 65 - 72 . 